\title{
Quantum Cryptography Based on Orthogonal States
}

\author{
Lior Goldenberg and Lev Vaidman
}

\author{
School of Physics and Astronomy \\ Raymond and Beverly Sackler Faculty of Exact Sciences \\ Tel Aviv University, Tel-Aviv 69978, Israel.
}

\begin{abstract}
All existing quantum cryptosystems use non-orthogonal states as the carriers of information. Non-orthogonal states cannot be cloned (duplicated) by an eavesdropper. In result, any eavesdropping attempt must introduce errors in the transmission, and therefore, can be detected by the legal users of the communication channel. Orthogonal states are not used in quantum cryptography, since they can be faithfully cloned without altering the transmitted data. In this Letter we present a cryptographic scheme based on orthogonal states, which also assures the detection of any eavesdropper.
\end{abstract}


A basic task in cryptography is exchanging a secret message between two users, traditionally called Alice and Bob, in a way that no other party can read it. The only known method to do this in a proven secure way is to use a 'one-time pad', which uses a previously shared secret information called a key. The key, a sequence of random bits, is used for encrypting the message. The encrypted message is completely confidential, even if transmitted via a public communication channel. Thus, the security of any key-based cryptographic method depends ultimately on the secrecy of the key. All existing classical key-distribution cryptosystems are not proven to be secure; their secrecy is based on computational complexity assumptions which sometimes turn out to be false. In particular, some existing cryptosystems can be broken (in principle) due to new developments [1] in quantum computation. On the other hand, the secrecy of quantum cryptosystems is guaranteed by the fundamental laws of quantum mechanics. Any intervention of an eavesdropper, Eve, must leave some trace which can be detected by the legal users of the communication channel.

In the last years many quantum cryptosystems were suggested. All these schemes use non-orthogonal states to encode the information. The first key-distribution scheme was presented by Bennett-Brassard [2] in 1984 (a variation of it was already tested experimentally [3]). In this scheme Alice transmits single photons polarized along one of four possible directions, $\uparrow, \leftrightarrow, \swarrow$ or $\nwarrow$. The first two are orthogonal in one basis and the other two are orthogonal in another basis. The encoding is as follows: Alice chooses, at random, one of the four states and sends it to Bob. It is agreed that the states $\leftrightarrow$ and $\nwarrow$ stand for bit value 0 , and the states $₫$ and $\swarrow$ stand for bit value 1 . Bob chooses, also at random, a basis, $\oplus$ or $\otimes$, and measures the polarization in that basis. If Alice and Bob choose the same basis, their results should be identical. If they choose different bases, their results are not correlated. By discussing over an insecure classical channel (which cannot be modified by an eavesdropper), Alice and Bob agree to discard all the cases where different bases were used (about half of the bits). The result should be two perfectly correlated strings, unless the transmission was disturbed. Any eavesdropping attempt must introduce errors in the transmission, since Eve does not know the polariza- 
tion of each photon. Whenever Alice and Bob measure in one basis and Eve in the other basis, the correlation of the strings is destroyed.

The encoding in quantum cryptography was based on non-orthogonal states since they cannot be cloned (duplicated) by an eavesdropper. Even an imperfect cloning attempt (intended to gain partial information) induces errors in the transmission, therefore it is detectable. In general, any two non-orthogonal states can be used for quantum cryptography, as shown by Bennett [4]. On the other hand, orthogonal states can be faithfully cloned, so that Eve can copy the data without being noticed. For these reasons it is generally believed that the use of non-orthogonal states is crucial in quantum cryptography. In this Letter we present a new quantum cryptosystem, in which data exchange between Alice and Bob is done using two orthogonal states, and yet, any eavesdropping attempt is detectable.

The security of our scheme is based on two novel ingredients. First, the orthogonal states sent by Alice are superpositions of two localized wavepackets. The wavepackets are not sent simultaneously towards Bob, but one of them is delayed for a fixed time and sent after the other. Second, the transmission time of each particle is random (and therefore, unknown to Eve). The tests performed by the users at the end of the communication allows the detection of an eavesdropper.

Let $|a\rangle$ and $|b\rangle$ be two localized wavepackets, which are sent from Alice to Bob along two separated channels. We shall take two orthogonal states $\left|\Psi_{0}\right\rangle$ and $\left|\Psi_{1}\right\rangle$, linear combinations of $|a\rangle$ and $|b\rangle$, to represent bit value '0' and bit value '1', respectively:

$$
\begin{aligned}
& \left|\Psi_{0}\right\rangle=1 / \sqrt{2}(|a\rangle+|b\rangle), \\
& \left|\Psi_{1}\right\rangle=1 / \sqrt{2}(|a\rangle-|b\rangle) .
\end{aligned}
$$

Alice sends to Bob either $\left|\Psi_{0}\right\rangle$ or $\left|\Psi_{1}\right\rangle$. The two localized wavepackets, $|a\rangle$ and $|b\rangle$, are not sent together, but wavepacket $|b\rangle$ is delayed for some time $\tau$. For simplicity, we choose $\tau$ to be larger than the traveling time of the particles from Alice to Bob, $\theta$. Thus, $|b\rangle$ starts traveling towards Bob only when $|a\rangle$ already has reached Bob, such that the two wavepackets are never found together in the transmission channels. 
In order to explain the idea behind the protocol, we shall consider a particular implementation of our scheme (the discussion assumes a noise-free transmission). The setup (Fig. (1) consists of a Mach-Zehnder interferometer with two storage rings, $S R_{1}$ and $S R_{2}$, of equal time delays. Alice can transmit a bit by sending a single particle either from the source $S_{0}$ (sending ' 0 ') or from the source $S_{1}$ (sending ' 1 '). The sending time $t_{s}$ is random, and it is registered by Alice for later use. The particle passes through the first beamsplitter $B S_{1}$ and evolves into a superposition of two localized wavepackets: $|a\rangle$, moving in the upper channel and $|b\rangle$, moving in the bottom channel. The particle coming from $S_{0}$ evolves into $\left|\Psi_{0}\right\rangle$ and the particle coming from $S_{1}$ evolves into $\left|\Psi_{1}\right\rangle$. The wavepacket $|b\rangle$ is delayed in the storage ring $S R_{1}$ while $|a\rangle$ is moving in the upper channel. When $|a\rangle$ arrives to the storage ring $S R_{2}$ at Bob's site, wavepacket $|b\rangle$ starts moving on the bottom channel towards Bob. During the flight-time of $|b\rangle$, wavepacket $|a\rangle$ is delayed in $S R_{2}$. Finally, the two wavepackets arrive simultaneously to the second beam-splitter $B S_{2}$ and interfere. A particle started in the state $\left|\Psi_{0}\right\rangle$ emerges at the detector $D_{0}$, and a particle started in the state $\left|\Psi_{1}\right\rangle$ emerges at the detector $D_{1}$. Bob, detecting the arriving particle, receives the bit sent by Alice: $D_{0}$ activated means ' 0 ' and $D_{1}$ activated means ' 1 '. In addition he registers the receiving time of the particle $t_{r}$.

Alice and Bob perform two tests (using a classical channel) in order to detect possible eavesdropping. First, they compare the sending time $t_{s}$ with the receiving time $t_{r}$ for each particle. Since the traveling time is $\theta$ and the delay time is $\tau$, there must be $t_{r}=t_{s}+\tau+\theta$. Second, they look for changes in the data by comparing a portion of the transmitted bits with the same portion of the received bits. If, for any checked bit, the timing is not respected or anti-correlated bits are found, the users learn about the intervention of Eve.

We will show that Eve, which has access to the channels but not to the sites of Alice and Bob, cannot extract any information without introducing detectable distortions in the transmission. The data is encoded in the relative phase between the two wavepackets $|a\rangle$ and $|b\rangle$. Therefore, the phase must be the same at $t_{s}$ and at $t_{r}$. In addition, the two wavepackets must arrive together to $B S_{2}$ at the correct time, otherwise a timing problem occurs. Any operation performed by Eve must obey these two requirements, or she will 
be exposed by the legal users.

Let us consider two times, $t_{1}$ and $t_{2}$. At $t_{1}$ the particle just left $B S_{1}$, so it is solely at Alice's site. At $t_{2}$ the particle is just before passing through $B S_{2}$ at Bob's site. If the particle is emitted from $S_{0}$, then at $t_{1}$ its state is $\left|\Psi_{0}\left(t_{1}\right)\right\rangle=1 / \sqrt{2}\left(\left|a\left(t_{1}\right)\right\rangle+\left|b\left(t_{1}\right)\right\rangle\right)$. If the particle is emitted from $S_{1}$, then at $t_{1}$ its state is $\left|\Psi_{1}\left(t_{1}\right)\right\rangle=1 / \sqrt{2}\left(\left|a\left(t_{1}\right)\right\rangle-\left|b\left(t_{1}\right)\right\rangle\right)$. In case that nothing disturbs the transmission (i.e. Eve is not present), the free time-evolution is

$$
\begin{aligned}
& \left|\Psi_{0}\left(t_{1}\right)\right\rangle \longrightarrow\left|\Psi_{0}\left(t_{2}\right)\right\rangle=1 / \sqrt{2}\left(\left|a\left(t_{2}\right)\right\rangle+\left|b\left(t_{2}\right)\right\rangle\right), \\
& \left|\Psi_{1}\left(t_{1}\right)\right\rangle \longrightarrow\left|\Psi_{1}\left(t_{2}\right)\right\rangle=1 / \sqrt{2}\left(\left|a\left(t_{2}\right)\right\rangle-\left|b\left(t_{2}\right)\right\rangle\right) .
\end{aligned}
$$

When Eve is present and she is trying to extract some information without being detected, the time-evolution must be such that $\left|\Psi_{0}\left(t_{1}\right)\right\rangle$ evolves to $\left|\Psi_{0}\left(t_{2}\right)\right\rangle$ and $\left|\Psi_{1}\left(t_{1}\right)\right\rangle$ evolves to $\left|\Psi_{1}\left(t_{2}\right)\right\rangle$ (if not, Bob will have a non-zero probability to receive inverted bits or to receive particles at incorrect times). Thus, the general form of the evolution from time $t_{1}$ to time $t_{2}$ must be:

$$
\begin{aligned}
& \left|\Psi_{0}\left(t_{1}\right)\right\rangle\left|\Phi\left(t_{1}\right)\right\rangle \longrightarrow\left|\Psi_{0}\left(t_{2}\right)\right\rangle\left|\Phi_{0}\left(t_{2}\right)\right\rangle, \\
& \left|\Psi_{1}\left(t_{1}\right)\right\rangle\left|\Phi\left(t_{1}\right)\right\rangle \longrightarrow\left|\Psi_{1}\left(t_{2}\right)\right\rangle\left|\Phi_{1}\left(t_{2}\right)\right\rangle,
\end{aligned}
$$

where $|\Phi(t)\rangle$ is the state of some auxiliary system used by Eve for extracting information. If $\left|\Phi_{0}\left(t_{2}\right)\right\rangle=\left|\Phi_{1}\left(t_{2}\right)\right\rangle$, no extraction of information is possible.

In protocols which use non-orthogonal quantum states for encryption, the time-evolution under eavesdropping must have the same form as eqs.(5) and (6). The security of these protocols, i.e. $\left|\Phi_{0}\left(t_{2}\right)\right\rangle=\left|\Phi_{1}\left(t_{2}\right)\right\rangle$, can be proven using the unitarity of quantum theory. When Eve is not present, from the free evolution (eqs.(3) and (田) we get $\left\langle\Psi_{1}\left(t_{1}\right) \mid \Psi_{0}\left(t_{1}\right)\right\rangle=$ $\left\langle\Psi_{1}\left(t_{2}\right) \mid \Psi_{0}\left(t_{2}\right)\right\rangle$. When Eve is present, from eqs.(5) and (6) we get $\left\langle\Psi_{1}\left(t_{1}\right) \mid \Psi_{0}\left(t_{1}\right)\right\rangle=$ $\left\langle\Psi_{1}\left(t_{2}\right) \mid \Psi_{0}\left(t_{2}\right)\right\rangle\left\langle\Phi_{1}\left(t_{2}\right) \mid \Phi_{0}\left(t_{2}\right)\right\rangle$. Combining these two results we find $\left|\Phi_{0}\left(t_{2}\right)\right\rangle=\left|\Phi_{1}\left(t_{2}\right)\right\rangle$. With orthogonal states, however, this proof fails, since $\left\langle\Psi_{1}\left(t_{1}\right) \mid \Psi_{0}\left(t_{1}\right)\right\rangle=0$. For this reason one might believe that quantum cryptography cannot rely on orthogonal states.

We shall prove now that our protocol is secure. Using the linearity of quantum theory, we consider the evolution of a particular superposition of $\left|\Psi_{0}\left(t_{1}\right)\right\rangle$ and $\left|\Psi_{1}\left(t_{1}\right)\right\rangle$. Consider 
at time $t_{1}$ a particle in the state $\left|b\left(t_{1}\right)\right\rangle=1 / \sqrt{2}\left(\left|\Psi_{0}\left(t_{1}\right)\right\rangle-\left|\Psi_{1}\left(t_{1}\right)\right\rangle\right)$. The time-evolution of $\left|b\left(t_{1}\right)\right\rangle\left|\Phi\left(t_{1}\right)\right\rangle$ is obtained from eqs.(5) and (6) (using also eqs.(3) and (4)):

$$
\left|b\left(t_{1}\right)\right\rangle\left|\Phi\left(t_{1}\right)\right\rangle \longrightarrow 1 / 2\left[\left|a\left(t_{2}\right)\right\rangle\left(\left|\Phi_{0}\left(t_{2}\right)\right\rangle-\left|\Phi_{1}\left(t_{2}\right)\right\rangle\right)+\left|b\left(t_{2}\right)\right\rangle\left(\left|\Phi_{0}\left(t_{2}\right)\right\rangle+\left|\Phi_{1}\left(t_{2}\right)\right\rangle\right)\right] .
$$

The last equation shows that, unless $\left|\Phi_{0}\left(t_{2}\right)\right\rangle=\left|\Phi_{1}\left(t_{2}\right)\right\rangle$, there is a non-zero probability to find the particle in the final state $\left|a\left(t_{2}\right)\right\rangle$. This, however, is impossible. A particle in the state $\left|a\left(t_{2}\right)\right\rangle$ is a particle which just emerged from the storage ring $S R_{2}$ (there is no other possibility). Since the delay time is $\tau$, at an earlier time than $t \equiv t_{2}-\tau$ the particle had to enter in Bob's site. At that time, a particle which started in the state $\left|b\left(t_{1}\right)\right\rangle$, as in eq.(7), is still captured in $S R_{1}$ at Alice's site. Such a particle enters in the bottom channel after time $t$, and then it is too late for Eve to send a dummy particle on the upper channel. She cannot send that particle at the correct time since she does not know it until the original wavepacket arrives. Thus, the state $\left|a\left(t_{2}\right)\right\rangle$ should not appear in the right-hand side of eq.(可), and therefore, $\left|\Phi_{0}\left(t_{2}\right)\right\rangle=\left|\Phi_{1}\left(t_{2}\right)\right\rangle$. This ends the proof.

We want to emphasize that the sending time cannot be publicly known, otherwise Eve could apply the following strategy: Using a replica of Alice's setup, she sends to Bob (at the correct time) a wavepacket $|b\rangle$ of a dummy particle, while waiting for Alice's particle. Using a replica of Bob's setup, she measures the later. Depending on the result of the measurement, she places a phase-shifter in front of the delayed wavepacket $|a\rangle$ of the dummy particle, in order to adjust the final interference. In this way Eve can extract the complete information without being exposed.

Since $\tau>\theta$, Eve has no access to $|a\rangle$ and to $|b\rangle$ together at any time. This seems to be a necessary requirement for a secure protocol, but it is not. If the communication is based on particles moving at the speed of light, it is enough to demand $\tau>\Delta t$, where $\Delta t$ is the accuracy of the time measurements of $t_{s}$ and $t_{r}$ (assuming very narrow wavepackets). The security in this case is proven in the same way: the state $\left|a\left(t_{2}\right)\right\rangle$ should not appear in eq.(17), since Eve gets wavepacket $|b\rangle$ too late for sending a dummy particle on the upper channel. Moreover, if we arrange a large distance between the two transmission channels (which requires large secure users' sites), we can use our procedure even without time 
delay. Any attempt of Eve to recombine the wavepackets in order to measure the phase, introduces an extra flight-time which will be detected by the users. However, now the security requires that Eve cannot use faster-than-light particles for eavesdropping. Thus, these versions of the protocol exceed the limits of non-relativistic quantum mechanics; they might be classified as "quantum-relativistic protocols" with orthogonal states.

In the previous discussion we have assumed ideal transmission conditions. In practice, any communication system is restricted by the limited efficiency of its components. The transmission is distorted by the noise of the channel, the losses and dark counts of the detectors, etc. Since errors from different sources are not necessarily distinguishable, Eve may obtain some information without being detected, as long as the amount of errors she introduces does not exceed the noise. Known methods of error correction and privacy amplification techniques can be included in a practical version of our protocol. The problems caused by losses and dark counts are automatically solved, due to the comparison between $t_{s}$ and $t_{r}$.

We shall raise some ideas related to the realization of our protocol in the laboratory. The first essential ingredient, random emission time, can be achieved very naturally using down-conversion crystal source of pairs of photons. In this way, the sending time of the photon is registered with very high efficiency and precision by a detector of the "idler" photon. The second ingredient, the time delay, can be achieved using an optical fiber loop. Probably, the most difficult part of the proposal is to have a Mach-Zehnder interferometer with a stable phase difference between its two (very long) arms. This problem can be avoided using one arm (an optical fiber) and two orthogonal polarizations as two quantum channels. In this setup wavepacket $|b\rangle$ leaves Alice's site when it is spatially delayed relative to wavepacket $|a\rangle$, and with a different polarization. In Bob's site, wavepacket $|a\rangle$ is delayed and its polarization direction is rotated, such that the two wavepackets finally interfere correctly.

Since there are some difficulties in an experiment with two polarization channels, a better way is sending the states with the same polarization, i.e. using a single channel. A modification of the setup in Fig. [ 1 allows the transmission of the wavepackets with the 
same polarization, but for the price of wasting a part of the photons [5]. A mirror and a beam-splitter added to Alice's site (after $S R_{1}$ ) can partially recombine the two channels into a single one. Similar beam-splitter and mirror added to Bob's site (before $S R_{2}$ ) can recover the two channels. As before, the users consider only photons which respect the timing requirement, but now a part of the sent photons are lost even if Eve is not present. Half of the photons are lost at Alice's site since they do not enter into the channel, and half of those which arrive to Bob's site are lost since they are detected at incorrect times. Thus, only $25 \%$ of the photons are usable, but this is good enough for key-distribution. The phase can be preserved more efficiently on a single channel, therefore this method might be practical for long-range transmission. One may be tempted to improve this proposal by introducing a setup which allows Bob to measure correctly all the transmitted photons. This can be done for the price of introducing uncertainty in the correlations between the sending and the receiving time of each photon, but then the method is not appropriate for our purpose (since Eve has time to get the signal and to resend it without being detected).

An advantage of using orthogonal states over non-orthogonal states is also related to the possibility of transmitting signals at long distances: orthogonal states can be 'enhanced' in intermediate stations, as classical signals are. Measuring a signal many times on the way decreases dramatically the amount of expected errors, due to the 'quantum Zeno effect'. The stations, however, have to be secure as the sites of Alice and Bob are.

Another advantage of our protocol (with two channels) over some other protocols (for example [6]) is that the bits are not random, but chosen by Alice, and that all the sent bits can be used. Therefore, the protocol is not restricted to key-distribution only - it can be used for sending the message directly [7]. Of course, Eve can read the message, but in an error-free channel she will be detected in time if Alice and Bob test the transmission frequently enough. The direct message transmission is possible not only on an error-free channel [5]. In a practical case (when noise is present), Alice and Bob agree in advance on the tolerable error rate and on the degrees of accuracy and secrecy they want to achieve. In order to transmit a message of some length $n$, Alice builds a longer string: some extra bits are used for estimating the error rate (hence, the maximal information leaked to Eve) 
and some for redundancy, which is used - via block-coding - to derive the $n$-bits message. The reliability of the $n$-bits message is assured by Shannon's channel coding theorem, (see [8]). At the end of the transmission, Alice tells Bob which bits were used for error estimation, and afterwards, the function used for block-coding. If Bob, estimating the error rate, detects Eve, he prevents publishing the block-coding function by informing Alice. Thus, the message is transmitted with an exponentially small probability of errors and exponentially small information leakage.

Let us conclude with a discussion of the title of our work. Strictly speaking, the set of all possible states sent by Alice is not a set of orthogonal states. Two states corresponding to identical bits, sent at two very close times, are not orthogonal. However, if the width of the wavepackets $|a\rangle$ and $|b\rangle$ is small enough, then the measure of mutual non-orthogonality is negligible. Moreover, we can replace the random sending times by random discreet sending times, and then, all the possible sent states will be mutually orthogonal. The previous proof assures the security of this procedure too. Note also, that in our basic method (with two channels) all the states corresponding to different bits are mutually orthogonal, and this is the relevant feature. Indeed, the issue of mutual orthogonality of just these states is essential for the security proof of protocols using non-orthogonal states.

The authors thank Tal Mor, Sandu Popescu, David DiVincenzo and Bruno Huttner for valuable comments. 


\section{References}

[1] P.W. Shor, in Proceedings of 35th Annual Symposium on Foundations of Computer Science (IEEE press, 1994), p. 124.

[2] C.H. Bennett and G. Brassard, in Proceedings of IEEE International Conference on Computers, Systems and Signal Processing, Bangalore, India (IEEE, N.Y., 1984), p. 175.

[3] C.H. Bennett, F. Bessette, G. Brassard, L. Salvail and J. Smolin, J. Cryptology 5, 3 (1992).

[4] C.H. Bennett, Phys. Rev. Lett. 68, 3121 (1992).

[5] T. Mor (private communication).

[6] A.K. Ekert, Phys. Rev. Lett. 67, 661 (1991).

[7] The same can be done in a modified version of Bennett-Brassard scheme [2], if Bob can delay his measurements until he is told the preparation basis of every photon.

[8] A.K. Ekert, B. Huttner, G.M. Palma and A. Peres, Phys. Rev. A 50, 1047 (1994).

\section{Figures Caption}

Figure 1: Cryptographic scheme based on a Mach-Zehnder interferometer. The device consists of two particle sources $S_{0}$ and $S_{1}$, a beam-splitter $B S_{1}$, two mirrors, two storage rings $S R_{1}$ and $S R_{2}$, a beam-splitter $B S_{2}$ and two detectors $D_{0}$ and $D_{1}$. The device is tuned in such a way that, if no eavesdropper is present, a particle emitted by $S_{0}\left(S_{1}\right)$ is finally detected by $D_{0}\left(D_{1}\right)$. 


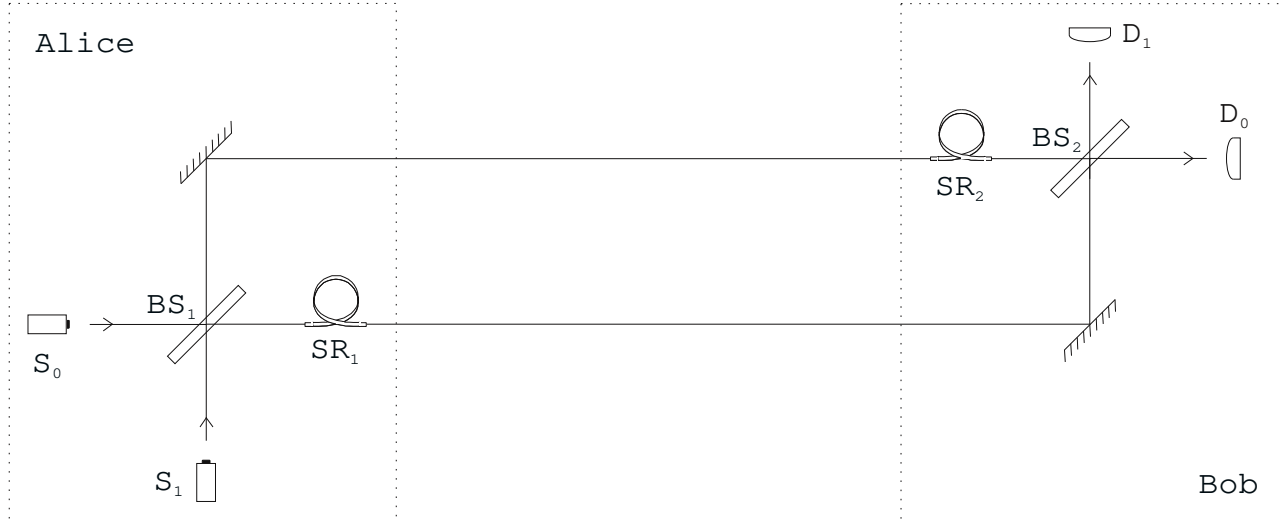


This figure "fig1-1.png" is available in "png" format from: http://arxiv.org/ps/quant-ph/9502021v1 